\title{
Observing sodiation process and achieving high efficiency of yolk-shell antimony@carbon rods
}

\author{
Xuming Yang ${ }^{1 \dagger}$, Yuanmin $\mathrm{Zhu}^{1 \dagger}$, Duojie $\mathrm{Wu}^{1}$, Kun Liu ${ }^{1}$, Haiyan Zhao ${ }^{1}$, Menghao $\mathrm{Li}^{1}$, Zhi Chang ${ }^{2}$ and \\ Meng $\mathrm{Gu}^{1 *}$
}

\begin{abstract}
As a promising anode material candidate for sodium-ion batteries, antimony ( $\mathrm{Sb}$ ) has attracted enormous research interest due to its high specific capacity and low sodiation voltage. However, its dramatic volume expansion upon sodiation adversely affects its cycle stability. We have developed an oxidation-coating-reduction strategy for fabricating yolk-shell Sb@C rods from commercially available Sb powder. In particular, the thermal reduction of vaporized $\mathrm{Sb}_{2} \mathrm{O}_{3}$ generates densely distributed $\mathrm{Sb}$ single atoms and clusters on the carbon shell. The sodiation process of the Sb@C sample was recorded through in situ transmission electron microscopy. Irregular expansion of $\mathrm{Sb}$ particles was observed, and it was also revealed that the carbon shell could deform with the expanded Sb particles. Beyond the intuitively understood advantage that internal voids can provide space for expansion of internal active materials, the deformability of carbon shells can add further ability to withstand the volume expansion. The two structural merits of the yolk-shell construction enable the Sb@C material to deliver an enhanced cycle performance. Its reversible capacity exceeds $620 \mathrm{~mA} \mathrm{~h} \mathrm{~g}^{-1}$ at $0.1 \mathrm{C}$, with an initial coulombic efficiency of up to $84.9 \%$, and about $95 \%$ of the capacity in the charging voltage profile is delivered below $1.0 \mathrm{~V}$ vs. $\mathrm{Na}^{+} / \mathrm{Na}$. These performance metrics are very promising for potential practical applications.
\end{abstract}

Keywords: sodium-ion batteries, antimony, in situ TEM, yolkshell, anode materials

\section{INTRODUCTION}

The ever-increasing growth of electricity generation from renewables has led to a tremendous demand for energy storage systems. Batteries with high cost and energy efficiency are a good fit for such applications. Sodium-ion batteries (SIBs), which have been developed as low-cost alternatives to the lithium-ion batteries (LIBs) that dominate the current market, are highly promising [1]. The commercialization of SIBs has been under discussion for years but has not yet been realized [2-4]. It is partially because the performance of electrode materials, both cathodes and anodes, has not been fully optimized to be satisfactory for practical applications. Remarkably, the lack of top-quality anode materials comparable to graphite in LIBs remains a major concern $[5,6]$.
The ideal anode should have a low sodiation voltage, large capacity, high cost-effectiveness, and easy processability. A wide variety of anode materials have been identified. However, those with a (de-)sodiation voltage below $1.0 \mathrm{~V} v s . \mathrm{Na}^{+} / \mathrm{Na}$ are mainly limited to the following types: sodium-metal anodes, hard carbon, phosphorous, and alloy-type anodes such as tin and antimony. All are considered promising candidates, with their own strengths and weaknesses. Metallic sodium is thermodynamically the ultimate choice for anodes, but the growth of dendrites during sodium plating can reduce capacity and cause safety issues. Phosphorous is very attractive due to its abundant resources and theoretical capacity of over $2000 \mathrm{~mA} \mathrm{~h} \mathrm{~g}^{-1}$. However, it suffers from low electrical conductivity, significant swelling upon sodiation, poor processability, and the most annoying points, producing highly flammable white phosphorous [7]. Hard carbon is the choice of anodes in prototypes of various SIBs for cost and performance analysis [8,9]. Its sodiation plateau, close to $0 \mathrm{~V} v s . \mathrm{Na}^{+} / \mathrm{Na}$, improves energy density, making hard carbon sensitive to premature discharging or unwanted sodium plating, especially at high current rates. In contrast, antimony $(\mathrm{Sb})$ with a sodiation voltage of about $0.6 \mathrm{~V}$ vs. $\mathrm{Na}^{+} / \mathrm{Na}$ has a much larger margin to compensate for polarization. In combination with its capacity of over $600 \mathrm{~mA} \mathrm{~h} \mathrm{~g}^{-1}, \mathrm{Sb}$ is likely to be eventually used for future practical applications.

The primal drawback of the $\mathrm{Sb}$ anode is dramatic volume expansion up to $390 \%$ after complete sodiation [10]. Repeated volume expansion and shrinkage can pulverize and disconnect active materials in the electrode, destroying solid electrolyte interphases (SEI). The most common strategy to address this problem is structural engineering [11]. One typical case is to encapsulate $\mathrm{Sb}$ particles within carbon fibers via electrospinning [12]. The as-prepared $\mathrm{Sb} / \mathrm{C}$ nanowires exhibit superior cycle stability, but the $\mathrm{Sb}$ content is only $54 \mathrm{wt} \%$. As $\mathrm{Sb}$ is less active than many transition metals, a templating method based on galvanostatic replacement reactions has been developed, which is unique for synthesizing anode materials $[13,14]$. For example, $\mathrm{Sb}$ nanotubes are produced from the replacement reactions between antimony chloride and copper nanowires [13,15]. It has also been reported that Sb-based binary or tertiary alloy compounds, for example, bismuth-antimony alloy, can enhance anodic performance. Their fabrication is usually coupled with intentional structural engineering [16,17]. Incorporating Sb into the carbon matrix is another effective method to immobilize $\mathrm{Sb}$

\footnotetext{
${ }^{1}$ Department of Materials Science and Engineering, Southern University of Science and Technology, Shenzhen 518055, China

${ }^{2}$ Energy Technology Research Institute, National Institute of Advanced Industrial Science and Technology (AIST), 1-1-1, Umezono, Tsukuba 305-8568, Japan

† These authors contributed equally to this work.

* Corresponding author (email: gum@sustech.edu.cn)
} 
components during battery operation [18]. For instance, Sb nanoparticles confined in chemically doped hollow carbon spheres can withstand 10,000 cycles at $20 \mathrm{~A} \mathrm{~g}^{-1}$ with a capacity of $331 \mathrm{~mA} \mathrm{~h} \mathrm{~g}^{-1}$ [19]. The reported performance metrics are highly impressive. However, it should be noted that the synthetic process is complex, the $\mathrm{Sb}$ content is low, areal capacity is small, and the initial coulombic efficiency (ICE) is not yet sufficient.

It is well known that elaborate $\mathrm{Sb} / \mathrm{C}$ structures help extend cycle life, but it is difficult to fabricate such structures with cheap materials and simple methods. From a practical point of view, it is important to reduce the carbon weight fraction and irreversible capacity in the initial cycle of $\mathrm{Sb} / \mathrm{C}$ composites. This needs to be emphasized but is often ignored. In this research, we developed a simple method to process commercially available $\mathrm{Sb}$ powders into yolk-shell Sb@C rods. More precisely, Sb particles are encapsulated in carbon tubes decorated with Sb single atoms and clusters. Based on in situ transmission electron microscopy (TEM), Sb particles are trapped inside the carbon tube during the sodiation process to ensure good cycle stability. The resulting sample has a high $\mathrm{Sb}$ content of over $90 \%$, providing good cycle and rate performance $\left(632 \mathrm{~mA} \mathrm{~h} \mathrm{~g}^{-1}\right.$ at $0.1 \mathrm{C}$ and $560 \mathrm{~mA} \mathrm{~h} \mathrm{~g}^{-1}$ at $1 \mathrm{C})$. The ICE can reach $84.9 \%$, which outperforms most values in the literature.

\section{EXPERIMENTAL SECTION}

\section{Synthesis of $\mathrm{Sb}_{2} \mathrm{O}_{3}$ rods}

$\mathrm{Sb}_{2} \mathrm{O}_{3}$ rods were obtained from the oxidation of commercially available $\mathrm{Sb}$ powder in ethylenediamine (EDA) and polyvinylpyrrolidone (PVP) aqueous solutions. Four gram of PVP $(\mathrm{Mw}=24,000)$ and $70 \mathrm{~mL}$ of EDA were dissolved in $700 \mathrm{~mL}$ of deionized water. Then, $4 \mathrm{~g}$ of Sb powder (1200 Mesh) was added under magnetic stirring until the suspension turned from black to milky white, and no black particles were observed on the bottom. Sediments $\left(\mathrm{Sb}_{2} \mathrm{O}_{3}\right.$ rods) were collected via centrifugation and washed with pure water.

\section{Synthesis of yolk-shell Sb@C rods}

Ultrasonication and magnetic stirring were used to disperse $1 \mathrm{~g}$ of $\mathrm{Sb}_{2} \mathrm{O}_{3}$ rods and $0.1 \mathrm{~g}$ of pyrrole in $300 \mathrm{~mL}$ of deionized water. Then, $10 \mathrm{~mL}$ of ammonium persulfate solution $\left(0.5 \mathrm{~mol} \mathrm{~L}^{-1}\right)$ was added, and the suspension turned slowly from white into black due to the polymerization of pyrrole. $\mathrm{Sb}_{2} \mathrm{O}_{3}$ was reduced to $\mathrm{Sb}$ by recovering the obtained black precipitate and annealing it in an $\mathrm{H}_{2} / \mathrm{Ar}$ mixed gas at $450^{\circ} \mathrm{C}$ for $4 \mathrm{~h}$.

\section{Characterization}

The morphology of the samples was identified using scanning electron microscopy (SEM, Tescam Mira 3) and TEM (Talos F200X G2). High-resolution images showing single $\mathrm{Sb}$ atoms were collected by a double aberration-corrected scanning TEM (Titan Themis G2). The diffraction pattern was recorded using an X-ray diffractometer (XRD, Bruker D8 Advance). Antimony content was determined using an inductively coupled plasma mass spectrometry (ICP-MS, Agilent 7700) machine.

\section{Electrochemical measurements}

The samples were mixed with conductive additive (acetylene black) and aqueous binder (sodium carboxymethyl cellulose, $\mathrm{NaCMC}$ ) solutions to form a viscous slurry. The weight ratio of the samples, acetylene black, and NaCMC was controlled at
$80: 10: 10$. The slurry was coated on copper foil and dried to fabricate electrode films, which were punched into disks with a diameter of $14 \mathrm{~mm}$. The mass loading of the active materials on a single electrode disk was $1.8-2.5 \mathrm{mg}$. After being completely dried, these disks were assembled into 2032-type coin cells using sodium metal foil as the counter electrode and glass fiber as the separator. The electrolyte was $1 \mathrm{~mol} \mathrm{~L}^{-1} \mathrm{NaClO}_{4}$ in ethylene carbonate (EC) and diethyl carbonate (PC) (volume ratio of EC: $\mathrm{PC}=50: 50$ ) containing 5\% fluoroethylene carbonate (FEC). Galvanostatic charge and discharge tests were performed on a Neware battery testing system. Cyclic voltammograms (CV) and electrochemical impedance spectroscopy (EIS) were recorded on an electrochemical workstation (Solartron 1470E MultiStat).

\section{In situ TEM test}

The yolk-shell Sb@C sample was attached to a gold grid and sodium metal with surface oxide layers on a tungsten tip. Both were mounted onto a scanning tunneling microscopy holder (Pico Femto FE-F20) and transferred into an environmental TEM (FEI G2). During the sodiation process, the Sb@C rod maintained contact with sodium, and a negative bias of $-2 \mathrm{~V}$ was applied.

\section{RESULTS AND DISCUSSION}

The easiest way to modify Sb for anode applications is downsizing and carbon coating. It is not allowed to create a hollow interior with these techniques. Unlike this intuitive method, we proposed an oxidation-reduction strategy that oxidizes $\mathrm{Sb}$ to $\mathrm{Sb}_{2} \mathrm{O}_{3}$ and then reduces $\mathrm{Sb}_{2} \mathrm{O}_{3}$ to $\mathrm{Sb}$. This reduces the size of $\mathrm{Sb}$ and can create void space due to the volume difference between $\mathrm{Sb}_{2} \mathrm{O}_{3}$ and $\mathrm{Sb}$ of equivalent $\mathrm{Sb}$ weight. The processing of commercial Sb powders into our target sample is schematically shown in Fig. 1. Using stepwise oxidation and polypyrrole (PPy) coating treatment, $\mathrm{Sb}$ powders are converted to core-shell $\mathrm{Sb}_{2} \mathrm{O}_{3} @ \mathrm{PPy}$ rods and subjected to thermal annealing in a reducing atmosphere. During the annealing process, the outer PPy shell is carbonized without noticeable structure changes, and the inner $\mathrm{Sb}_{2} \mathrm{O}_{3}$ rods are reduced to $\mathrm{Sb}$ particles trapped in the carbon shell. Thus, yolk-shell Sb@C rods are obtained by such an oxidizing-coating-reducing strategy. It should be noted that $\mathrm{Sb}_{2} \mathrm{O}_{3}$ vaporization is possible even at high temperatures, and the reduction of $\mathrm{Sb}_{2} \mathrm{O}_{3}$ vapor can produce $\mathrm{Sb}$ inside or outside the carbon shell.

Commercially available $\mathrm{Sb}$ powders used in this research are irregular particles at the scale of tens of micrometers (Fig. S1). They were gradually oxidized to $\mathrm{Sb}_{2} \mathrm{O}_{3}$ when dispersed in an aqueous EDA solution containing a certain amount of PVP. This was reflected in the color change of the resulting suspension from black into milky white. EDA was used to catalyze the reactions between $\mathrm{Sb}$ powders and oxygen in the solution, and PVP plays the role of structure-directing reagent [20]. After complete oxidation, the collected white precipitate was rods with lengths ranging from below $1 \mu \mathrm{m}$ to over $10 \mu \mathrm{m}$, as shown in Fig. 2a. According to the XRD pattern, the precipitate was confirmed to be pure $\mathrm{Sb}_{2} \mathrm{O}_{3}$ (Fig. S2). The suspension of $\mathrm{Sb}_{2} \mathrm{O}_{3}$ rods in water is white, and it slowly turns black after adding pyrrole and an oxidizing reagent (ammonium persulfate). The black precipitate obtained was also rod-shaped (Fig. 2b) and uniformly coated with a thin layer of PPy (Fig. S3). After thermal annealing in a mixed $\mathrm{Ar} / \mathrm{H}_{2}$ atmosphere, the recorded XRD pattern was perfectly matched with a Sb phase (PDF\#85-1324), 


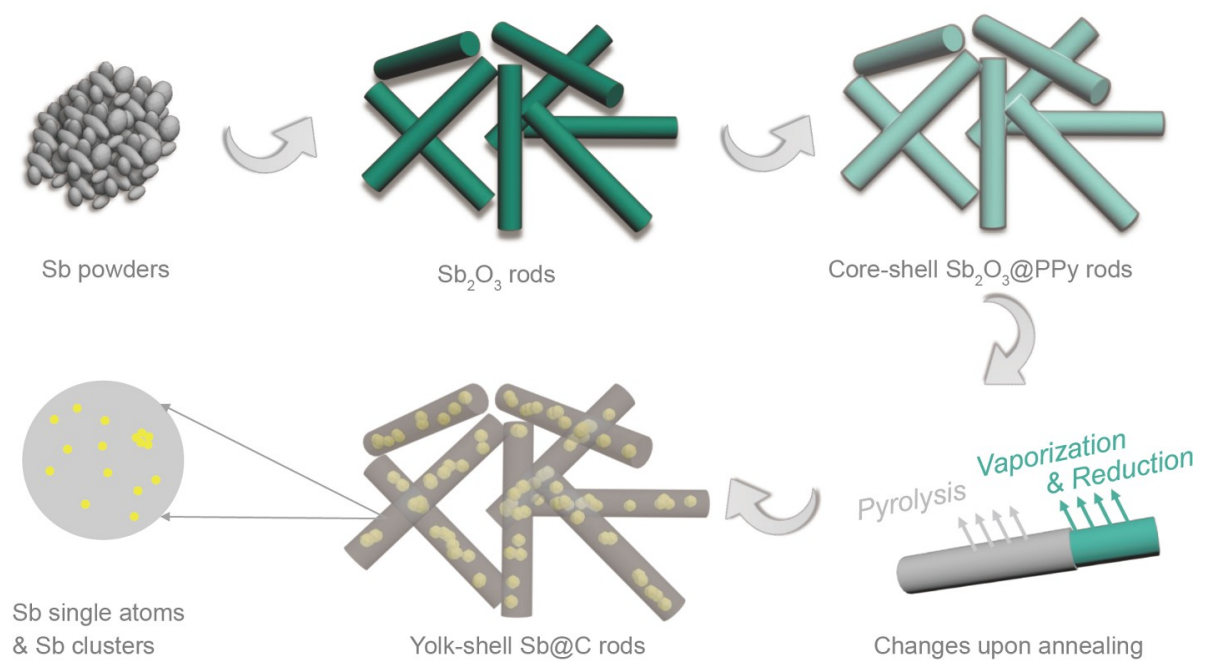

Figure 1 Schematic illustration of the oxidizing-coating-reducing strategy of processing Sb powders into yolk-shell Sb@C rods.
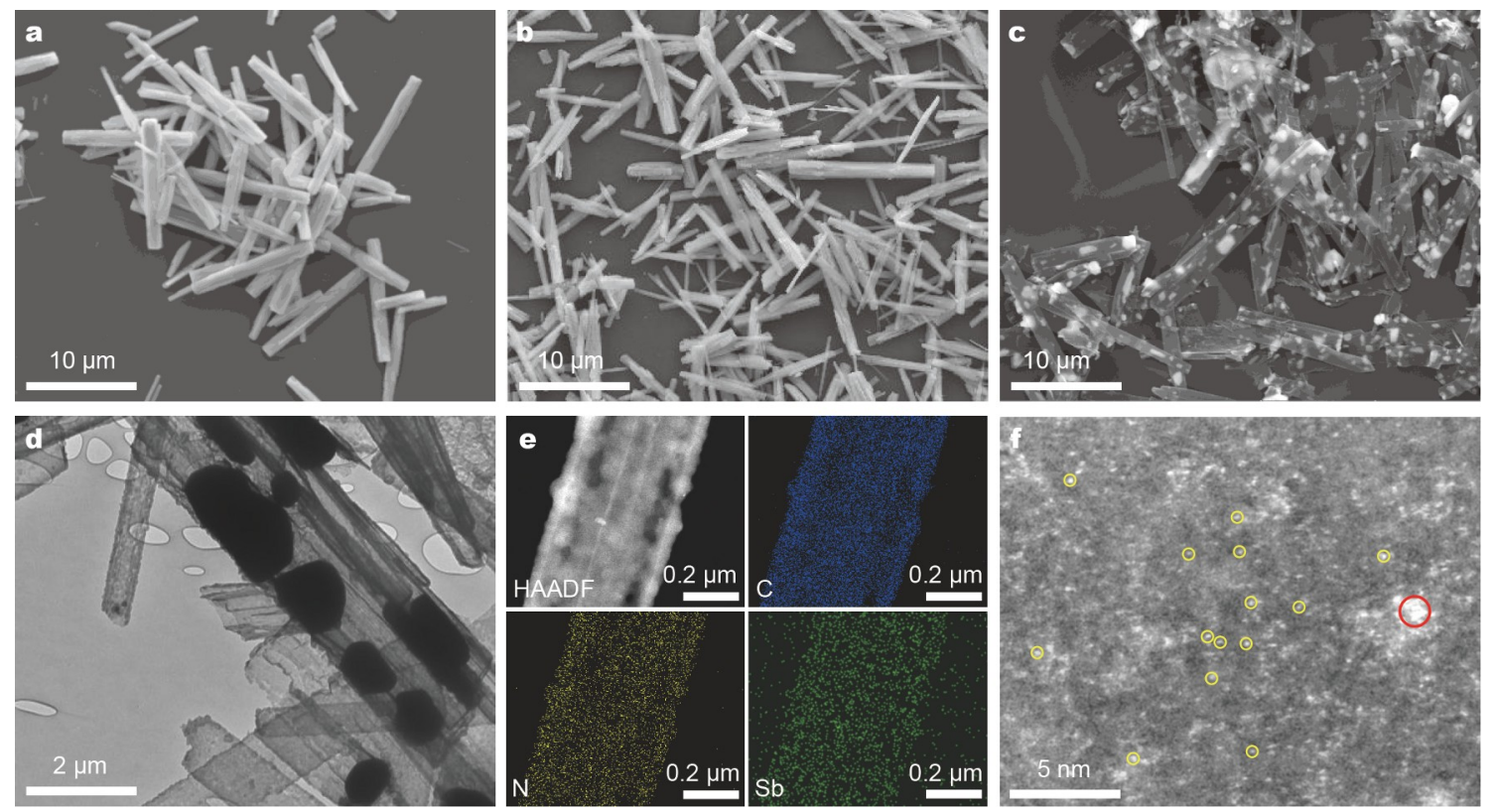

Figure 2 SEM images of (a) $\mathrm{Sb}_{2} \mathrm{O}_{3}$, (b) $\mathrm{Sb}_{2} \mathrm{O}_{3} @ P P y$, and (c) yolk-shell Sb@C rods; (d) TEM image and (e) high-angle annular dark field (HAADF) image and the corresponding energy dispersive X-ray spectroscopy (EDS) mapping results at a selected area of yolk-shell Sb@C rods; (f) high-resolution HAADF image of the carbon shell showing Sb single atoms (yellow circles) and clusters (red circle).

indicating a complete conversion from $\mathrm{Sb}_{2} \mathrm{O}_{3}$ to $\mathrm{Sb}$. As shown in Fig. 2c, the annealed sample is composed of fine $\mathrm{Sb}$ particles inside a carbon shell derived from the PPy coating layers. The $\mathrm{Sb}$ content of the Sb@C composite was 91\% based on ICP-MS measurement.

The molar volumes of $\mathrm{Sb}$ and $\mathrm{SbO}_{3 / 2}$ were calculated to be 17.5 and $25.3 \mathrm{~cm}^{3} \mathrm{~mol}^{-1}$, respectively. Thus, Sb particles theoretically take up 69\% (namely 17.5/25.3) of the inner space of the carbon shell. Nevertheless, the total volume occupied by $\mathrm{Sb}$ particles is obviously less than $69 \%$, and some rods are even empty, as shown in Fig. 2d. This is because the sublimation of $\mathrm{Sb}_{2} \mathrm{O}_{3}$ occurs at the annealing temperature, allowing the outward diffusion of $\mathrm{Sb}_{2} \mathrm{O}_{3}$. The elemental mapping of selected regions with no visible particles revealed that $\mathrm{Sb}$ is also present (Fig. 2e). Many single atoms of $\mathrm{Sb}$ and some $\mathrm{Sb}$ clusters are observed in the high-resolution images taken by the double aberration-corrected scanning transmission electron microscope (Fig. 2f). These are probably produced from the reduction of vaporized $\mathrm{Sb}_{2} \mathrm{O}_{3}$.

Producing the yolk-shell Sb@C has at least two benefits. Voids inside the carbon shell can accommodate the specific volume expansion of Sb during sodiation, and carbon shells can support the structure by trapping sodiation products inside. To demonstrate the two benefits, in situ TEM experiments allowed real-time observation of the sodiation process. Specifically, we built a nanobattery with the configuration shown in Fig. 3a. Therefore, the sodiation process can be initiated by applying a voltage bias using a potentiostat, and the TEM imaging can be performed throughout the process.

The expansion process of $\mathrm{Sb}$ particles inside the carbon shell 

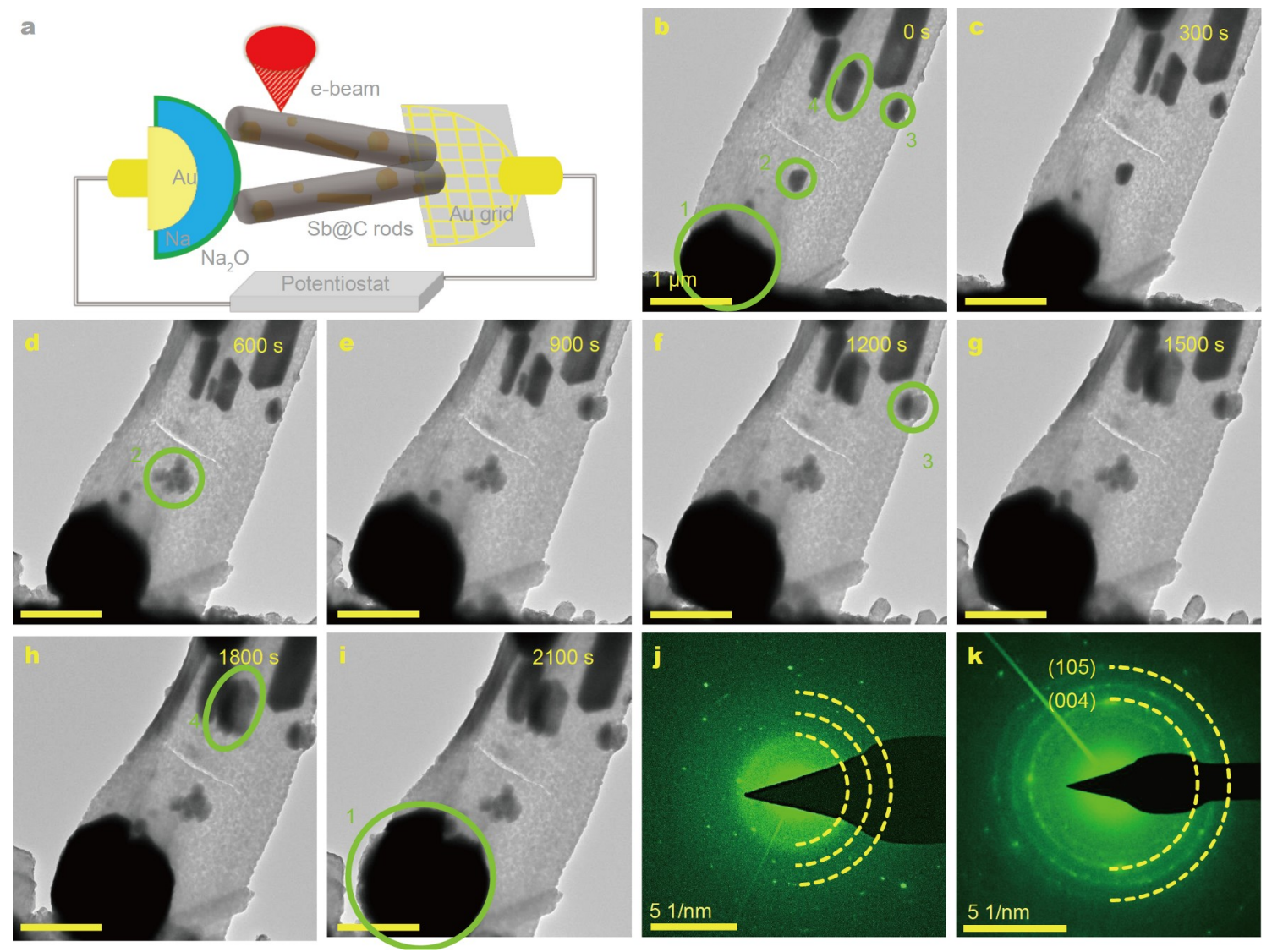

Figure 3 (a) The schematic of the cell setup for in situ TEM tests; (b-i) TEM images of a yolk-shell Sb@C rod at different sodiation times; electronic diffraction patterns of a yolk-shell Sb@C rod at the (j) initial and (k) final states.

(see Movie S1) was recorded, and eight selected frames are shown in Fig. 3b-i. Four particles marked in Fig. 3b indicate that they have undergone volume expansion in various ways. As sodium moved along the carbon shell, the sodiation of particles close to metal sodium began preferentially. Particle 1, which was close to metal sodium, became much larger than it was in the original state after $300 \mathrm{~s}$. In such conditions, the other particles basically remained unchanged. It grew slowly and became more spherical along the sodiation process. It should be noted that the radius of the carbon shell increased with the expansion of Particle 1, but no cracks were observed. This means that the carbon shell is deformable rather than rigid, providing the yolk-shell Sb@C structure with the ability to accommodate volume expansion beyond the inner void space. Particle 2, which was much smaller, exploded during the sodiation (Fig. 3d) and did not change after $600 \mathrm{~s}$, indicating that the sodiation was completed within $300 \mathrm{~s}$. The sodiation process for Particle 3 with a similar size of Particle 2 was also finished within $300 \mathrm{~s}$, but no branches occurred after sodiation. Particle 4 was an elongated rod whose expansion was mainly along the radial direction. Differences in structure evolutions suggest that the expansion of $\mathrm{Sb}$ particles is in random directions.

In the selected area electron diffraction (SAED) patterns at the initial and end states, circles corresponding to the plane spacing of $\mathrm{Sb}$ (PDF\#85-1324) and $\mathrm{Na}_{3} \mathrm{Sb}$ (PDF\#74-1162) are drawn, respectively (Fig. 3j, k). As expected, the scattered bright spots are arranged on the circles. The identification of $\mathrm{Na}_{3} \mathrm{Sb}$ after sodiation is consistent with the literature, indicating that $\mathrm{Sb}$ is sodiated to $\mathrm{Na}_{3} \mathrm{Sb}$. Based on these results, the theoretical expansion after sodiation is calculated to be $390 \%$, and if the structure evolution is conformal, the size change will be $157 \%$ (namely $390 \%{ }^{1 / 3}$ ). As these particles are irregular, it is impractical to quantify the volume expansion. However, it is still reasonable to conclude that the observed expansion is in good agreement with the theoretical value of the trend. This undoubtedly shows that $\mathrm{Sb}$ particles are trapped in the carbon shell throughout the sodiation process. Therefore, outstanding cycle stability can be expected.

Fig. 4a shows the CV of the yolk-shell Sb@C rods at $0.1 \mathrm{mV} \mathrm{s}^{-1}$. Sodiation in the initial cycle is a direct conversion from $\mathrm{Sb}$ to $\mathrm{Na}_{3} \mathrm{Sb}$, as reflected in the only cathodic peak at $0.27 \mathrm{~V}$ vs. $\mathrm{Na}^{+} / \mathrm{Na}$. However, three peaks are identified in the second cycle, corresponding to a multi-step sodiation process as previously reported [14]. Based on these results, the voltage profile at $0.1 \mathrm{C}\left(1 \mathrm{C}\right.$ is defined as $0.6 \mathrm{~A} \mathrm{~g}^{-1}$ for simplicity), shown in Fig. $4 \mathrm{~b}$, features one discharge plateau in the first cycle but three in the second. As the first plateau covers about one-third of the total capacity, an intermediate phase of $\mathrm{NaSb}$ is proposed. Based on the formation enthalpy data from the Materials Project (www.materialsproject.org), the theoretical average voltage values of $\mathrm{Sb}$ into $\mathrm{Na}_{3} \mathrm{Sb}, \mathrm{Sb}$ into $\mathrm{NaSb}$, and $\mathrm{NaSb}$ into $\mathrm{Na}_{3} \mathrm{Sb}$ are evaluated to be $0.58,0.65$, and $0.54 \mathrm{~V} v s$. $\mathrm{Na}^{+} / \mathrm{Na}$, respectively. In consideration of voltage polarization in real batteries, the data presented are in good agreement with theoretical results. The 

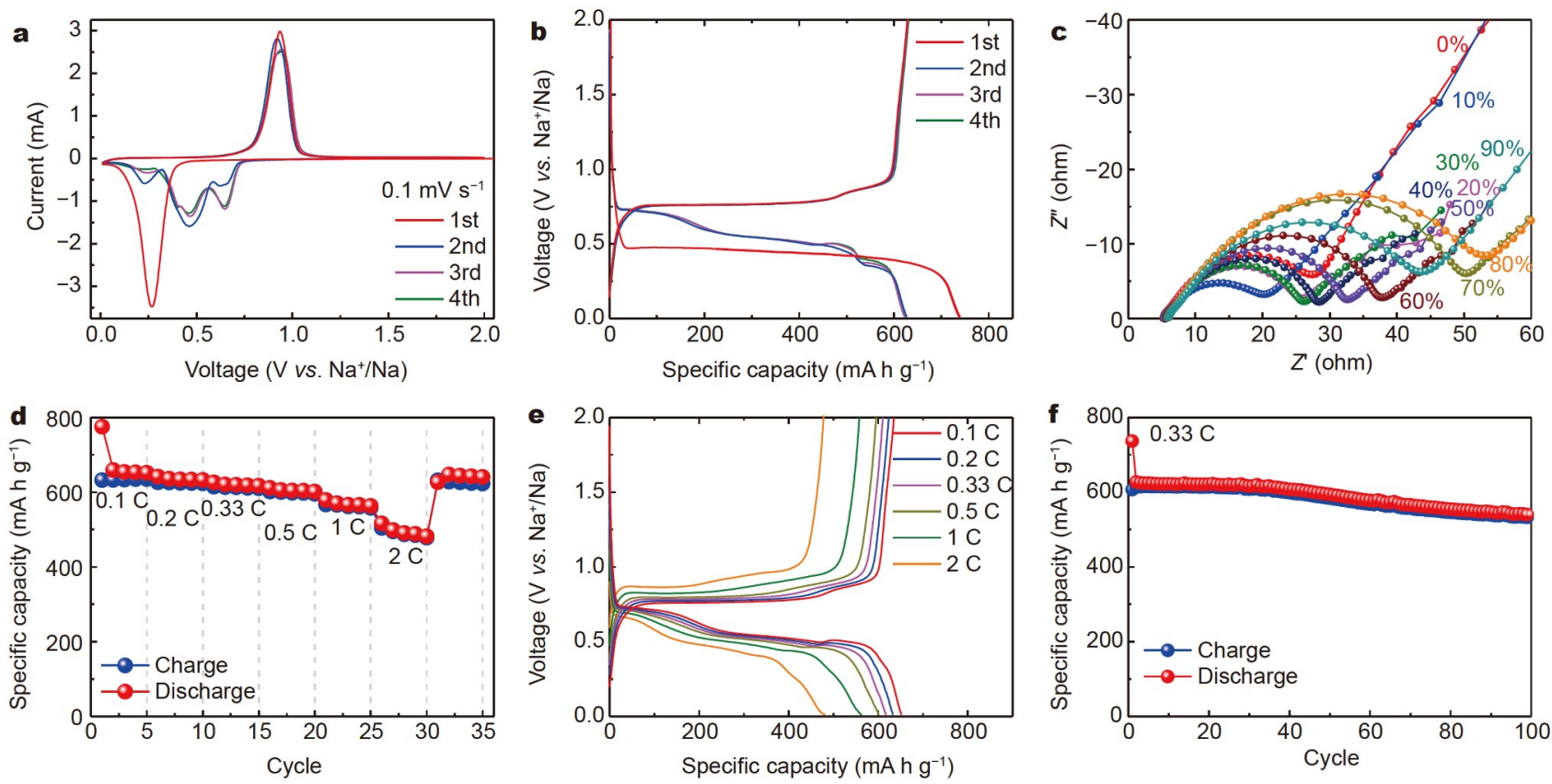

Figure 4 Battery testing results of the yolk-shell Sb@C. (a) CV at $0.1 \mathrm{mV} \mathrm{s}^{-1}$; (b) voltage profile at $0.1 \mathrm{C}$; (c) EIS at various states of charge; (d) rate performance and (e) the corresponding voltage profiles from 0.1 to $2 \mathrm{C}$; (f) cycle performance at $0.33 \mathrm{C}$.

CV curve in the 3rd cycle overlaps with the CV curve in the 4th cycle. The voltage profiles are essentially identical after the initial cycle, indicating the high reversibility of the anode reactions.

In contrast, the commercially available Sb powders are highly unstable during the battery test, and the specific capacity decreases to below $50 \mathrm{~mA} \mathrm{~h} \mathrm{~g}^{-1}$ in only five cycles (Fig. S4). As shown in Fig. 4c, during stepwise sodiation, the EIS depends on the discharge/sodiation depth. From the changes in the semicircle (Fig. S5), it is roughly speculated that the sodiation kinetics should be inversely proportional to the degree of sodiation [21].

In the rate performance test (Fig. $4 \mathrm{~d}$ ), as the rate current increases from 0.1 to $0.5 \mathrm{C}$, the capacity decreases only from 632 to $601 \mathrm{~mA} \mathrm{~h} \mathrm{~g}^{-1}$. Also, from the voltage profiles shown in Fig. $4 \mathrm{e}$, the polarization during charging or discharging does not deteriorate significantly. As the current increases to $2 \mathrm{C}$, a capacity reduction becomes apparent. From our point of view, this is because dramatic changes in volume over a short period of time are out of control. Thus, fast discharging should be avoided. In the cycle test at $0.33 \mathrm{C}$, the capacity reduction in the first 40 cycles is negligible, and the capacity retention after 100 cycles is about $87 \%$. The reversible capacity at $0.1 \mathrm{C}$ exceeds $620 \mathrm{~mA} \mathrm{~h} \mathrm{~g}^{-1}$, the ICE is as high as $84.9 \%$, the average voltage of the entire charging process is calculated to be $0.81 \mathrm{~V} v s . \mathrm{Na}^{+} / \mathrm{Na}$ (Fig. S6), and about $95 \%$ of the capacity is below $1.0 \mathrm{~V} v s . \mathrm{Na}^{+} / \mathrm{Na}$.

These performance metrics are very promising for the development of SIB anode materials. As shown in Table S1, the introduction of a carbon matrix generally improves $\mathrm{Sb}$ utilization and cycle stability. High Sb content is very important to promise a specific capacity advantage over other candidate anode materials such as hard carbon. This study is one of the few cases with an Sb content of over $90 \%$ and a reversible capacity of over $600 \mathrm{~mA} \mathrm{~h} \mathrm{~g}^{-1}$. Compared with the two pioneering studies on $\mathrm{Sb}$ anodes $[12,22]$, it is necessary to improve the ICE, which is currently approaching the documented records. Such high effi- ciency in the initial cycle indicates low consumption of $\mathrm{Na}$ in SEI generation and high reversibility of the alloying reaction between $\mathrm{Na}$ and $\mathrm{Sb}$. It should also be pointed out that the cycle stability in high-rate or long-term tests needs to be further improved. The coulombic efficiency after the initial cycle (mainly in the range of 98.5 to 99.1 ) is decent in the literature but not sufficient for practical applications.

\section{CONCLUSIONS}

In summary, a simple method of processing commercially available Sb powders into yolk-shell Sb@C rods was developed. $\mathrm{Sb}$ powders were oxidized to $\mathrm{Sb}_{2} \mathrm{O}_{3}$ rods under the catalysis of EDA and processed into Sb@C rods after PPy coating and thermal reduction. The void space in such structures originates from the difference in molar volume between $\mathrm{Sb}$ and $\mathrm{Sb}_{2} \mathrm{O}_{3}$. On the other hand, the sublimation of $\mathrm{Sb}_{2} \mathrm{O}_{3}$ at high annealing temperatures also contributes to some extent. It is worth mentioning that the reduction of vaporized $\mathrm{Sb}_{2} \mathrm{O}_{3}$ gives rise to the dense distribution of $\mathrm{Sb}$ single particles on the carbon shell. This is probably of interest to scientists working on single-atom catalysts.

We conducted in situ TEM by assembling the Sb@C sample and metal sodium into a nanobattery. Although the volume expansion of $\mathrm{Sb}$ particles is irregular, it is found that the particles are trapped inside the carbon shell during the entire sodiation process. Based on the real-time observations, two benefits of the yolk-shell structures become apparent: the internal void space can accommodate volume expansion, and the deformable carbon shell can be expanded to provide additional space. Electrochemical measurements demonstrate excellent reversibility between sodiation and de-sodiation. As a result, several exciting performance metrics have been reported, including an ICE of up to $84.9 \%$, specific capacities over $620 \mathrm{~mA} \mathrm{~h} \mathrm{~g}^{-1}$, and about $95 \%$ capacity below $1.0 \mathrm{~V} v s$. $\mathrm{Na}^{+} / \mathrm{Na}$ at a low current rate. We believe that these results will encourage battery researchers to re- 
examine the practicality of antimony anodes and inspire those who work with similar battery systems, such as potassium-ion batteries, in which $\mathrm{Sb}$ anodes are also applicable [23].

\section{Received 13 April 2021; accepted 10 June 2021;}

published online 23 August 2021

1 Grey CP, Tarascon JM. Sustainability and in situ monitoring in battery development. Nat Mater, 2017, 16: 45-56

2 Deng J, Luo WB, Chou SL, et al. Sodium-ion batteries: From academic research to practical commercialization. Adv Energy Mater, 2017, 8: 1701428

3 Chen L, Fiore M, Wang JE, et al. Readiness level of sodium-ion battery technology: A materials review. Adv Sustain Syst, 2018, 2: 1700153

4 Bauer A, Song J, Vail S, et al. The scale-up and commercialization of nonaqueous Na-ion battery technologies. Adv Energy Mater, 2018, 8: 1702869

5 Yang X, Rogach AL. Anodes and sodium-free cathodes in sodium ion batteries. Adv Energy Mater, 2020, 10: 2000288

6 Massé RC, Uchaker E, Cao G. Beyond Li-ion: Electrode materials for sodium- and magnesium-ion batteries. Sci China Mater, 2015, 58: 715766

7 Xia Q, Li W, Miao Z, et al. Phosphorus and phosphide nanomaterials for sodium-ion batteries. Nano Res, 2017, 10: 4055-4081

8 Vaalma C, Buchholz D, Weil M, et al. A cost and resource analysis of sodium-ion batteries. Nat Rev Mater, 2018, 3: 18013

9 Peters J, Peña Cruz A, Weil M. Exploring the economic potential of sodium-ion batteries. Batteries, 2019, 5: 10

10 Tan H, Chen D, Rui X, et al. Peering into alloy anodes for sodium-ion batteries: Current trends, challenges, and opportunities. Adv Funct Mater, 2019, 29: 1808745

11 Li W, Zeng L, Wu Y, et al. Nanostructured electrode materials for lithium-ion and sodium-ion batteries via electrospinning. Sci China Mater, 2016, 59: 287-321

$12 \mathrm{Zhu} \mathrm{Y}, \mathrm{Han} \mathrm{X}, \mathrm{Xu} \mathrm{Y}$, et al. Electrospun $\mathrm{Sb} / \mathrm{C}$ fibers for a stable and fast sodium-ion battery anode. ACS Nano, 2013, 7: 6378-6386

13 Liu Y, Zhou B, Liu S, et al. Galvanic replacement synthesis of highly uniform Sb nanotubes: Reaction mechanism and enhanced sodium storage performance. ACS Nano, 2019, 13: 5885-5892

14 Hou H, Jing M, Yang Y, et al. Sodium/lithium storage behavior of antimony hollow nanospheres for rechargeable batteries. ACS Appl Mater Interfaces, 2014, 6: 16189-16196

15 Yang K, Tang J, Liu Y, et al. Controllable synthesis of peapod-like Sb@C and corn-like C@Sb nanotubes for sodium storage. ACS Nano, 2020, 14: 5728-5737

16 Farbod B, Cui K, Kalisvaart WP, et al. Anodes for sodium ion batteries based on tin-germanium-antimony alloys. ACS Nano, 2014, 8: 44154429

17 Guo S, Li H, Lu Y, et al. Lattice softening enables highly reversible sodium storage in anti-pulverization $\mathrm{Bi}-\mathrm{Sb}$ alloy/carbon nanofibers. Energy Storage Mater, 2020, 27: 270-278

$18 \mathrm{Wu} \mathrm{C}$, Shen $\mathrm{L}$, Chen S, et al. Top-down synthesis of interconnected two-dimensional carbon/antimony hybrids as advanced anodes for sodium storage. Energy Storage Mater, 2018, 10: 122-129

19 Chen B, Qin H, Li K, et al. Yolk-shelled Sb@C nanoconfined nitrogen/ sulfur Co-doped 3D porous carbon microspheres for sodium-ion battery anode with ultralong high-rate cycling. Nano Energy, 2019, 66: 104133

20 Deng $\mathrm{Z}$, Tang $\mathrm{F}$, Chen $\mathrm{D}$, et al. A simple solution route to singlecrystalline $\mathrm{Sb}_{2} \mathrm{O}_{3}$ nanowires with rectangular cross sections. J Phys Chem B, 2006, 110: 18225-18230

21 Yang X, Rogach AL. Electrochemical techniques in battery research: A tutorial for nonelectrochemists. Adv Energy Mater, 2019, 9: 1900747

$22 \mathrm{Wu} \mathrm{L}, \mathrm{Hu} \mathrm{X}$, Qian J, et al. Sb-C nanofibers with long cycle life as an anode material for high-performance sodium-ion batteries. Energy Environ Sci, 2014, 7: 323-328

23 Cao K, Liu H, Jia Y, et al. Flexible antimony@carbon integrated anode for high-performance potassium-ion battery. Adv Mater Technol, 2020, 5: 2000199

Acknowledgements This work was supported by the National Natural Science Foundation of China (21802065 and 12004156), Shenzhen DRC Project [2018]1433, Shenzhen Science and Technology Program (KQTD20190929173815000), Shenzhen Basic Research Fund (JCYJ20190809181601639), Guangdong Innovative and Entrepreneurial Research Team Program (2019ZT08C044). The microscopy charaterization was performed at the Pico Center at SUSTech that receives support from the Presidential Fund and Development and Reform Commission of Shenzhen Municipality. Yang X acknowledges SUSTech Presidential Postdoctoral Fellowship.

Author contributions $\mathrm{Gu} \mathrm{M}$ conceived the idea and led the project. Yang $\mathrm{X}$ designed, prepared and tested the samples. Zhu Y conducted the in situ TEM experiments and the analysis of relevant data. $\mathrm{Wu} \mathrm{D}$ and Zhao $\mathrm{H}$ were involved in the electrochemical tests. Liu K and Li M helped to characterize the samples. Yang X wrote the paper under the supervision of $\mathrm{Gu} \mathrm{M}$. Chang $\mathrm{Z}$ helped to improve the manuscript. All authors contributed to the general discussion.

Conflict of interest The authors declare that they have no conflict of interest.

Supplementary information Supporting data are available in the online version of the paper.

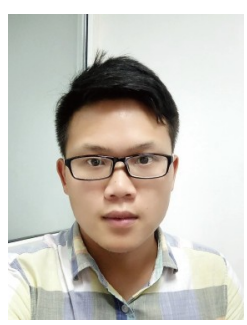

Xuming Yang received his Bachelor's and Master's degrees from the Central South University in 2013 and 2016, respectively. He got his $\mathrm{PhD}$ degree in materials science from the City University of Hong Kong in 2019. After graduation, he joined Prof. Meng Gu's group as a postdoctoral fellow. His research focuses on battery-related materials and their operating mechanisms.

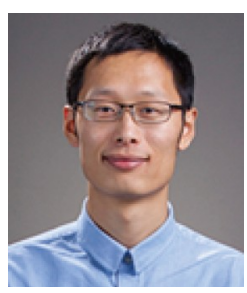

Yuanmin Zhu received his $\mathrm{PhD}$ degree in materials science from the University of Science and Technology Beijing in 2016. Then, he joined Beijing Electron Microscopy Center at Tsinghua University as a postdoc researcher. In 2018, He joined the Southern University of Science and Technology as a research assistant professor. His research interests include the atomic and electronic structures of materials surface and interface based on the aberration-corrected transmission electron microscopy, micro-dynamic behaviors of the energy materials using in situ environmental-TEM.

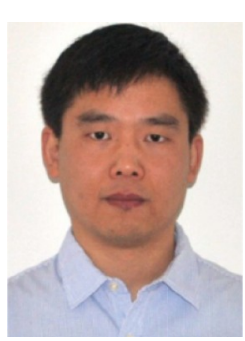

Meng Gu received his $\mathrm{PhD}$ degree in materials science from the University of California, Davis. He is now a professor at the Southern University of Science and Technology. His research focuses on the development of better catalysts, various rechargeable batteries, and advanced electron microscopy characterization and analysis. 


\section{中空核壳型锑碳材料的钠化过程观测与高效率储钠}

杨旭明 ${ }^{1 \dagger}$, 祝远民 ${ }^{1 \dagger}$, 吴多杰 ${ }^{1}$, 刘坤 ${ }^{1}$, 赵海燕 ${ }^{1}$, 李梦浩 ${ }^{1}$, 常智 ${ }^{2}$, 谷猛 ${ }^{*}$

摘要 锑因其高容量、低电压等优点成为备受关注的钠离子电池负极 材料, 但钠化过程中巨大的体积膨胀使其难以实现稳定的循环. 本文提 出了一个氧化-包覆-还原的策略, 将不适合直接用作负极的商业化锑粉 加工成中空核壳型棒状锑碳复合材料. 氧化锑受热升华还原的过程中, 在碳层上形成了密集分布的锑单原子. 利用原位环境电镜, 将该材料构 建成微观纳米电池, 实现了钠化过程的实时观测. 发现铇在钠化过程中 发生不规则膨胀, 碳层可随锑膨胀产生相应变形. 碳层内部的原有空间 以及碳层向外扩张的能力, 均有利于维持碳层对铮在钠化膨胀过程中 的包覆效果. 在电池测试中, 该材料在 $0.1 \mathrm{C}$ 下的可逆比容量超过 $620 \mathrm{~mA} \mathrm{~h} \mathrm{~g}^{-1}$, 首圈的库伦效率高达 $84.9 \%$ (显著高于文献报道的水平), 以及在 $1 \mathrm{~V} v s . \mathrm{Na}^{+} / \mathrm{Na}$ 的电位以下可完成 $95 \%$ 的充电容量. 该材料优异 的电池性能说明中空核壳型结构设计可用于开发实用型锑基钠电负极 材料. 